\title{
Effect of radiotherapy on resin composite adaptation to the cavity wall and elastic modulus of dentin
}

\author{
Takako Yoshikawa, DDS, PhD (1), Makoto Arakawa, DDS, PhD (2), and Junji Tagami, \\ DDS, PhD (1)
}

(1) Department of Cariology and Operative Dentistry, Division of Oral Health Sciences, Graduate School of Medical and Dental Sciences, Tokyo Medical and Dental University, Tokyo, Japan, and (2) Department of Dental Hygiene, Chiba Prefectural University of Health Sciences, Chiba, Japan

Purpose: This study aimed to evaluate the effects of 60 Gy $\gamma$-ray irradiation on the adaptation of resin composite restorations using two-step and one-step self-etching systems.

Materials and Methods: One-half of bovine incisors were irradiated with 60 Gy $\gamma$-ray. Flat dentin surfaces were prepared on labial side. Cylindrical cavities were prepared on flat dentin surfaces. Cavities were restored with a two-step self-etching system Clearfil SE Bond or a one-step self-etching system Clearfil tri-S Bond adhesive, followed by Clearfil AP-X composite. The composites were light-cured with $600 \mathrm{~mW} / \mathrm{cm}^{2}$ for $40 \mathrm{~s}$. One-half of the specimens were thermocycled for 5,000 cycles. Dye penetration tests around the restorations were performed.

Results: Irradiation with 60 Gy $\gamma$-ray significantly decreased resin composite adaptation for the nonthermocycled Clearfil SE Bond $(p<0.05)$. Clearfil tri-S Bond showed significantly decreased cavity wall adaptation compared with Clearfil SE Bond, regardless of the number of thermal cycling and $\gamma$-ray irradiation ( $p$ $<0.05)$. Irradiation with 60 Gy $\gamma$-ray significantly decreased nanohardness and elastic modulus of the dentin $(p<$ $0.05)$.

Conclusion: Irradiation with 60 Gy $\gamma$-ray significantly decreased resin composite adaptation for the non-thermocycled Clearfil SE bond. Further, nanohardness and elastic modulus of the dentin significantly decreased after the irradiation.

(Asian Pac J Dent 2019; 19: 39-44.)

Key Words: adaptation, dye penetration, elastic modulus, $\gamma$-ray irradiation, microhardness, resin composite

\section{Introduction}

The aging population in society has witnessed increased incidence of tooth wear and cancer. Patients with head and neck cancer require radiotherapy in these areas [1]. Orofacial tissues, including the salivary glands, mucous membranes, taste buds, bone, and teeth are affected by radiation [2-4]. Radiation caries can be attributed to head or neck radiotherapy [5] in the cervical area of the tooth [6]. Tooth wear and radiation caries cannot be treated using metal inlays commonly used for conventional Black's cavities. They can only be restored with resin composites using a direct bonding technique to conserve the tooth structure.

Radiation causes decrease in the saliva production resulting from salivary gland lesion [7] and changes in the microbial flora [8]. Most orofacial complications are dependent on radiation dose, and severe side-effects occur when doses are greater than $45 \mathrm{~Gy}$ [9]. Irradiation of $60 \mathrm{~Gy} \gamma$-ray is the total absorbed dose in one course of radiotherapy in patients with head or neck cancer. Irradiation by 60 Gy $\gamma$-ray significantly decreased the ultimate tensile strength [10] and microhardness of the dentin [11]; however, 60 Gy $\gamma$-ray significantly increased the microhardness of superficial enamel [11]. Irradiation with 70 Gy $\gamma$-ray significantly decreased the shear bond strength of the bovine dentinoenamel junction and could cause some damage to the biophysical properties [12].

Resin composite restoration with a direct bonding technique has been indicated in cancer patients before or after irradiation. However, resin composite adaptation to the irradiated tooth substrate has seldom been reported. The effect of $\gamma$-ray irradiation on dentin remains unclear. It is hypothesized that $60 \mathrm{~Gy} \gamma$-ray irradiation will decrease resin composite adaptation to the dentin cavity wall, dentin nanohardness, and elastic modulus. 


\section{Materials and Methods}

\section{Specimen preparation}

The materials, components, manufacturers, batch numbers, and bonding procedures used in this study are listed in Table 1. An experimental quartz-tungsten halogen light-curing unit (GC, Tokyo, Japan) connected to a slide regulator (Type SD-135, Matsunaga Mfg., Yokohama, Japan) was used in this study. This light-curing unit included a control system for lamp voltage and adjustable light intensity, which was measured using a curing radiometer (model 100, Demetron Research, Danbury, CT, USA). Forty erupted intact bovine lower incisors, stored frozen after extraction, were employed in this study. One-half of the bovine incisors were irradiated with 60 Gy $\gamma$-rays using a cobalt 60 therapeutic machine (RCR-120; Toshiba, Tokyo, Japan). The labial enamel was ground using a model trimmer under running water to expose a superficial flat dentin surface and finished with a wet 600 -grit $\mathrm{SiC}$ paper. Cylindrical cavities of $1.5 \mathrm{~mm}$ depth, $3 \mathrm{~mm}$ diameter, and a $\mathrm{C}$-factor of 3 were prepared on the fiat dentin surfaces of each tooth using a diamond point (\#CR30, ISO No. 068 030, GC) under copious air-water spray. Each of the nine cavities was treated with a two-step self-etching system Clearfil SE Bond (SE, Kuraray Noritake Dental, Tokyo, Japan) or a one-step self-etching system Clearfil tri-S Bond (TS, Kuraray Noritake Dental). After the adhesive was cured, the cavities were bulk-filled with Clearfil AP-X resin composite (shade A3, Kuraray Noritake Dental). The resin composite was light-cured with an intensity of $600 \mathrm{~mW} / \mathrm{cm}^{2}$ for $40 \mathrm{~s}$.

Table 1 Study materials

\begin{tabular}{|c|c|c|c|}
\hline Material/Manufacturer & Components $^{\mathrm{a}}$ & Batch No. & Bonding Instruction $^{\mathrm{b}}$ \\
\hline \multirow[t]{2}{*}{$\begin{array}{l}\text { Clearfil SE Bond } \\
\text { Kuraray Noritake } \\
\text { Dental, Tokyo, Japan }\end{array}$} & $\begin{array}{l}\text { Primer: MDP, HEMA, hydrophilic aliphatic } \\
\text { dimethacrylates, colloidal silica, photoinitiator, } \\
\text { accelerators, water }\end{array}$ & $00761 \mathrm{~A}$ & $\mathrm{a}(20 \mathrm{~s}), \mathrm{b}, \mathrm{c}, \mathrm{d}, \mathrm{e}(10 \mathrm{~s})$ \\
\hline & $\begin{array}{l}\text { Bond: MDP, HEMA, Bis-GMA, hydrophobic } \\
\text { aliphatic methacrylate, microfiller (colloidal silica), } \\
\text { photoinitiator, initiator, accelerators, others }\end{array}$ & 01099A & \\
\hline $\begin{array}{l}\text { Clearfil tri-S Bond } \\
\text { Kuraray Noritake } \\
\text { Dental }\end{array}$ & $\begin{array}{l}\text { Bond: MDP, HEMA, Bis-GMA, photoinitiator, water, } \\
\text { ethanol, silanated colloidal silica }\end{array}$ & $00083 \mathrm{~A}$ & c $(20 \mathrm{~s}), \mathrm{b}, \mathrm{e}(10 \mathrm{~s})$ \\
\hline $\begin{array}{l}\text { Clearfil AP-X, shade A3 } \\
\text { Kuraray Noritake } \\
\text { Dental }\end{array}$ & $\begin{array}{l}\text { silanated barium glass filler, silanated silica filler, } \\
\text { silanated colloidal silica, Bis-GMA, TEGDMA, } \\
\text { photoinitiator, catalyst, accelerator, pigments, others } \\
\text { Filler load: } 84.5 \mathrm{wt} \%\end{array}$ & $01151 \mathrm{~A}$ & \\
\hline
\end{tabular}

\footnotetext{
${ }^{\text {a }}$ Abbreviations: Bis-GMA, bisphenol A-glycidyl methacrylate; MDP, 10-methacryloyloxydecyl dihydrogen phosphate; HEMA, 2-hydroxyethylmethacrylate; TEGDMA, triethyleneglycol dimethacrylate

${ }^{\mathrm{b}}$ Procedures: a, apply primer; b, dry with gently air-blowing; c, apply adhesive; d, gently air-blow; e, light-cure
}

\section{Evaluation of marginal sealing and cavity wall adaptation}

After completion of light curing, the specimens were stored in the dark for $24 \mathrm{~h}$ in water maintained at $37^{\circ} \mathrm{C}$. One-half of the specimens were subsequently thermocycled for 5,000 cycles between at $5^{\circ} \mathrm{C}$ and $55^{\circ} \mathrm{C}$ with a 30 $\mathrm{s}$ dwell time. The dye penetration test was used to determine the degree of adaptation to the cavity margins and walls. The test was performed by placing a $1.0 \%$ acid red propylene glycol solution (Caries Detector, Kuraray Noritake Dental) at the margin of restoration for $5 \mathrm{~s}$, followed by rinsing with water and gentle blow-drying. The extent of dye penetration was observed with a stereo-microscope (20× magnification). A photographic record of each specimen was acquired at this stage.

The specimens were then longitudinally cut in half using a diamond saw microtome (77 model, Bronwill 
Scientific, Rochester, NY, USA) under running water, the dye was reapplied to the sections, and images were acquired to observe the gaps. In these images, the length of dye penetration along the cavity margins and walls was measured using a digitizer (KD 4300 model, Graphtec, Tokyo, Japan). The degree of marginal leakage was defined as the length of dye penetration, which was measured as a percent of the total length of the cavity margin. Dye penetration along the cavity walls was calculated as a percent of the total cavity wall length. This was referred to as the cavity-wall gap formation. The dye penetration test scores were compared using the Kruskal-Wallis and Mann-Whitney U tests at a significance level of 5\%.

\section{Nanohardness and elastic modulus}

Each of four teeth that were irradiated and non-irradiated were embedded using epoxy resin. The labial enamel was ground using a model trimmer under running water to expose the superficial flat dentin surface and finished with a wet diamond paste. Nanohardness and complex Young's modulus were measured at the dentin surface of each non-irradiated and irradiated specimen. Each specimen of four nanohardness and elastic modulus were measured using a nanoindentation tester (ENT-1100, Elionix, Hachioji, Japan) with a load of $0.049 \mathrm{~N}$ and $12 \mathrm{~s}$ dwell time. Nanohardness and elastic modulus results were analyzed using the Bonferroni/Dunn test at a significance level of $5 \%$.

\section{Results}

The results of marginal leakage and cavity-wall gap formation are shown in Table 2. The group of Clearfil SE Bond with 0 cycle showed no dye penetration on margin, regardless of $\gamma$-ray irradiation. $\gamma$-ray irradiation significantly decreased the resin composite adaptation to the cavity wall of Clearfil SE Bond with 0 cycle group $(p<0.05)$. There was no significant difference between the irradiated and non-irradiated groups with 5,000 cycles thermal cycling on marginal sealing and cavity wall adaptation for Clearfil SE Bond $(p>0.05)$. However, 5,000 cycles thermal cycling significantly increased resin composite adaptation to the cavity wall compared with the group of 0 cycle thermal cycling of the $\gamma$-ray irradiated for Clearfil SE Bond $(p<0.05)$.

Table 2 The degree of marginal leakage and cavity-wall gap formation

\begin{tabular}{|c|c|c|c|c|c|}
\hline \multirow{2}{*}{$\begin{array}{l}\text { Number of } \\
\text { thermal cycling }\end{array}$} & \multirow{2}{*}{$\begin{array}{l}\text { Adhesive } \\
\text { system }\end{array}$} & \multicolumn{2}{|c|}{ Marginal leakage (\%) } & \multicolumn{2}{|c|}{ Cavity-wall gap formation (\%) } \\
\hline & & Non-irradiated & Irradiated & Non-irradiated & Irradiated \\
\hline \multirow[t]{2}{*}{0} & SE & 0 & 0 & $7.0(10.5)^{\mathrm{a}, \mathrm{A}}$ & $19.9(10.3)^{\mathrm{a}, \mathrm{B}}$ \\
\hline & TS & 0 & 0 & $29.5(8.3)$ & $43.7(20.8)^{\mathrm{C}}$ \\
\hline \multirow[t]{2}{*}{5,000} & SE & $1.1(3.2)$ & $0.9(2.9)$ & 0 & $2.4(4.9)^{\mathrm{B}}$ \\
\hline & TS & $1.1(3.2)$ & $0.7(2.2)$ & $27.2(19.5)^{\mathrm{A}}$ & $28.4(14.0)^{\mathrm{C}}$ \\
\hline
\end{tabular}

Intergroup data designated with the same lower-case superscript letters for each non-irradiated or irradiated dentin substrate are significantly different $(p<0.05)$. Intergroup data designated with the same upper-case superscript letters for each thermal cycling number are significantly different $(p<0.05)$. SD in parentheses, Intragroup data connected by a vertical line for adhesive type are significantly different $(p<0.05)$.

The group of Clearfil tri-S Bond with 0 cycle group showed no dye penetration on margin, regardless of $\gamma$-ray irradiation. There was no significant difference between the irradiated and non-irradiated groups with 5,000 cycles thermal cycling on marginal sealing and cavity wall adaptation for Clearfil tri-S Bond $(p>0.05)$. Clearfil tri-S Bond significantly decreased resin composite adaptation to the cavity wall compared with Clearfil SE Bond, regardless of $\gamma$-ray irradiation and thermal cycling number $(p<0.05)$.

There was no significant difference between 0 cycle and 5,000 cycles on marginal sealing of the 
non-irradiated group for both Clearfil SE Bond and Clearfil tri-S Bond ( $p>0.05)$. However, thermal cycling at 5,000 cycles significantly increased resin composite adaptation to the cavity wall compared with the irradiated group with 0 cycle for both Clearfil SE Bond and Clearfil tri-S Bond $(p<0.05)$.

The results of nanohardness and elastic modulus of dentin are shown in Table 3. Nanohardness and elastic modulus of the irradiated dentin were significantly lower than that of non-irradiated dentin $(p<0.05)$.

Table 3 Nanohardness and elastic modulus of non-irradiated and y-ray irradiated dentin

\begin{tabular}{ccc}
\hline & Nanohardness $(\mathrm{GPa})$ & Elastic Modulus $(\mathrm{GPa})$ \\
\hline Non-irradiated & $0.56(0.02) \mid$ & $25.9(1.3) \mid$ \\
Irradiated & $0.53(0.05) \mid$ & $21.4(3.2) \mid$ \\
\hline Intragroup data connected by a vertical line are significantly different $(p<0.05)$. SD in parentheses
\end{tabular}

\section{Discussion}

Irradiation with $60 \mathrm{~Gy} \gamma$-ray significantly decreased the resin composite adaptation to the cavity wall compared with non-irradiation for Clearfil SE Bond of non-thermal cycling group. Irradiation with 60 Gy $\gamma$-ray significantly decreased nanohardness and elastic modulus of the irradiated dentin compared with the non-irradiated dentin. This finding supported previous report that 60 Gy $\gamma$-ray significantly reduced Knoop hardness of the human dentin [11]. Yoshikawa et al. have already reported that 60 Gy $\gamma$-ray irradiation showed some effect to dentin morphology [13]. It was reported that 60 Gy $\gamma$-ray irradiation significantly decreased the ultimate tensile strength [10], and shear bond strength of resin composite to the dentin [14]. Moreover, 60 Gy $\gamma$-ray $[11,13]$ or 70 Gy $\gamma$-ray [12] irradiation caused some damage to the dentin collagen. Therefore, 60 Gy $\gamma$-ray irradiation decreased resin composite adaptation to the cavity wall for Clearfil SE Bond.

Thermal cycling at 5,000 cycles significantly increased resin composite adaptation to the cavity wall compared with the irradiated group with 0 cycle for both two-step self-etching system Clearfil SE Bond and one-step self-etching system Clearfil tri-S Bond. It was reported that resin composite bond strength to dentin of 1 min after light curing was lower than that of $24 \mathrm{~h}$ after light curing [15]. Therefore, thermal cycling increased composite adaptation to the cavity wall for irradiated group because of accelerated adhesive polymerization. It is thought that bond strength of both one-step and two-step self-etching systems were affected by $\gamma$-ray irradiation damage to the dentin at the early stage of bonding.

The two-step self-etching system Clearfil SE Bond showed excellent resin composite adaptation to the dentin cavity wall. However, one-step self-etching system Clearfil tri-S Bond showed significantly decreased resin composite adaptation to the dentin cavity wall compared with two-step self-etching system Clearfil SE Bond regardless of $\gamma$-ray irradiation and thermal cycling number. The mechanical properties of each component of resin-dentin bond complex (resin composite, adhesive layer, hybrid layer, and underlying dentin), are believed to play significant roles in bond stability that may contribute to bond durability [16,17]. It was reported that Clearfil SE Bond had significantly less water sorption compared with other adhesives [18]. During the thermal cycling procedure, the specimens were subjected to temperature changes and water immersion. Lower water sorption, hence less water molecules within the polymer matrix, helped to maintain the mechanical properties of dental resins over time. Thus, this lower water sorption behavior may account for the more durable bond [19,20]. Moreover, Yoshikawa et al. have already reported that the two-step self-etching system Clearfil SE Bond showed high microtensile bond strength on deep flat (thin RDT) dentin [21] and on Class I dentin cavity floor 
and wall as compared with one-step self-etching system Clearfil tri-S Bond [22,23]. Thus, Clearfil SE Bond showed excellent resin composite adaptation to the dentin cavity wall.

Conversely, one-step self-etching systems are more hydrophilic and water absorbent than two-step systems [18]. This water sorption plasticize polymers and increases solubility while decreasing the modulus of elasticity and the mechanical properties of the polymers [24]. Therefore, the one-step self-etching system Clearfil tri-S Bond showed lower adaptation to the cavity wall.

It was reported that $60 \mathrm{~Gy} \gamma$-ray irradiation showed no effect on the resin composite tensile bond strengths for the human flat dentin with four different dentin bonding agents using divided irradiation for 6 weeks [25]. Further research is required to confirm the clinically divided $\gamma$-ray irradiation effect of resin composite adaptation and bonding to the tooth substrate.

Irradiation with 60 Gy $\gamma$-ray significantly decreased resin composite adaptation for the non-thermocycled Clearfil SE bond. Further, nanohardness and elastic modulus of the dentin significantly decreased after the irradiation.

\section{Acknowledgment}

This work was supported by Grant-in-Aid for Scientific Research (B) No. 17390513 from the Ministry of Education, Culture, Sports, Science and Technology, Japan and Grant-in-Aid for Scientific Research (C) No. 16K11543 from the Japan Society for the Promotion of Science.

\section{Conflict of Interest}

There are no conflicts of interest to declare.

\section{References}

1. Lyons A. Current concept in the management of oral cancer. Dent Update 2006; 33: 538-9.

2. Carl W. Oral and dental care for cancer patients receiving radiation and chemotherapy. Quintessence Int Dent Dig 1981; 9: 861-9.

3. Kielbassa AM, Hinkelbein W, Hellwig E, Meyer-Lückel H. Radiation-related damage to dentition. Lancet Oncol 2006; 7: 326-35.

4. Fränzel W. Effect of tumor therapeutic irradiation on the mechanical properties of teeth tissue. Z Med Phys 2006; 16 : 148-54.

5. Craddock HL. Treatment and maintenance of a dental patient with 'radiation caries'. Dent Update 2006; 33: 462-4.

6. Pyykönen H, Malmström M, Oikarinen VJ, Salmo M, Vehkalahti N. Late effect of radiation treatment of tongue and floor-of mouth cancer on the dentition, saliva section, mucous membranes and the lower jaw. Int J Oral Maxillofac Surg 1986; 15: 401-9.

7. Frank RM, Herdly J, Phillipe E. Acquir dental defects and salivary gland lesions after irradiation for carcinoma. J Am Dent Assoc 1965; 70: 868-83.

8. Brown LR, Dreizen S, Handler S, Johnston DA. Effect of radiation-induced xerostomia on human oral microflora. J Dent Res 1975; $54: 740-50$

9. Bruce R, Rothwell BR. Prevention and treatment of the orofacial complications of radiotherapy. J Am Dent Assoc 1987; 114: 316- 22.

10. Soares CJ, Castro CG, Neiva NA, Soares PV, Santos-Filho PCF, Naves LZ, et al. Effect of gamma irradiation on ultimate tensile strength of enamel and dentin. J Dent Res 2010; 89: 159-64.

11. Gonçalves LMN, Palma-Dibb RG, Paula-Silva HF, Oliveira HF, Nelson-Filho P, Silva LA, et al. Radiation therapy alters microhardness and microstructure of enamel and dentin of permanent human teeth. J Dent 2014; 4: 986-92.

12. Pioch T, Golfels D, Staehle HJ. An experimental study of the stability of irradiated teeth in the region of the dentinoenamel junction. Endod Dent Traumatol 1992; 8: 241-4.

13. Yoshikawa T, Sadr A, Tagami J. Effect of radiotherapy on resin composite bond strength and adaptation to dentin. Asian Pac J Dent 2017; 17: 49-54.

14. Bulucu B, Yesilyurt C, Çakır S, Meydan AD. Influence of radiation on bond strength. J Adhes Dent 2006; 8: $217-21$.

15. Burrow MF, Tagami J, Negishi T, Nikaido T, Hosoda H. Early tensile bond strengths of several enamel and dentin bonding systems. J Dent Res 1994; 73: 522-8.

16. Sano H, Takatsu T, Ciucchi B, Russell CM, Pashley DH. Tensile properties of resin-infiltrated demineralized human dentin. J Dent Res 1995; 74: 1093-102.

17. Takahashi A, Sato Y, Uno S, Pereira PN, Sano H. Effects of mechanical properties of adhesive resins on bond strength to dentin. Dent Mater 2002; 18: 263-8.

18. Ito S, Hashimoto M, Wadgaonkar B, Svizero N, Carvalho RM, Yiu C, et al. Effects of resin hydrophilicity on water sorption and changes in modulus of elasticity. Biomaterials 2005; 26: 6449-59.

19. Miyazaki M, Sato M, Onose H, Moore BK. Influence of thermal cycling on dentin bond strength of two-step bonding systems. Am J Dent 1998; 11: 118-22

20. Koshiro K, Inoue S, Tanaka T, Koase K, Fujita M, Hashimoto M, et al. In vivo degradation of resindentin bonds produced by a self-etch vs. a total-etch adhesive system. Eur J Oral Sci 2004; 112: 368-75. 
21. Yoshikawa $\mathrm{T}$, Wattanawongpitak N, Cho E, Tagami J. Effect of remaining dentin thickness on bond strength of various adhesive systems to dentin. Dent Mater J 2012; 31: 1033-8.

22. Yoshikawa T, Sadr A, Tagami J. Effects of C-factor on bond strength to floor and wall dentin. Dent Mater J 2016; 35: 918-22.

23. Yoshikawa T, Sadr A, Arakawa M, Tagami J. Bond Strength of resin composites to cavity floor and cavity wall dentin. Asia Pac J Dent 2016; 16: 23-8.

24. Bastioli C, Romano G, Migliaresi C. Water sorption and mechanical properties of dental composites. Biomaterials 1990; 11: 219-23.

25. Gernhardt CR, Kielbassa AM, Hahn P, Schaller HG. Tensile bond strengths of four different dentin adhesives on irradiated and non irradiated human dentin in vitro. J Oral Rehabil 2001; 28: 814-20.

Dr. Takako Yoshikawa

Correspondence to:

Department of Cariology and Operative Dentistry, Division of Oral Health Sciences,

Graduate School of Medical and Dental Sciences, Tokyo Medical and Dental University (TMDU)

1-5-45, Yushima, Bunkyo-ku, Tokyo 113-8549, Japan

Fax: +81-3-5803-0195 E-mail: yoshikawa.ope@tmd.ac.jp

Copyright (C2019 by the Asian Pacific Journal of Dentistry.

Accepted April 19, 2019.

Online ISSN 2185-3487, Print ISSN 2185-3479 